\title{
COMPARISON THEOREMS ON THE OSCILLATION OF A CLASS OF NEUTRAL DIFFERENCE EQUATIONS WITH CONTINUOUS VARIABLES
}

\author{
BAŞAK KARPUZ AND ÖZKAN ÖCALAN
}

\begin{abstract}
In this paper, we introduce an iterative method to study oscillatory properties of delay difference equations of the following form $\nabla_{\alpha}[x(t)-r(t) x(t-\kappa)]+p(t) x(t-\tau)-q(t) x(t-\sigma)=0, \quad t \geq t_{0}$, where $t_{0} \in \mathbb{R}, t$ varies in the real interval $\left[t_{0}, \infty\right), \alpha>0, \kappa, \tau, \sigma \geq 0$, $r \in C\left(\left[t_{0}-\alpha, \infty\right), \mathbb{R}^{+}\right), p, q \in C\left(\left[t_{0}, \infty\right), \mathbb{R}^{+}\right)$and $\nabla_{\alpha} x(t)=x(t)-$ $x(t-\alpha)$ for $t \geq t_{0}$.
\end{abstract}

\section{Introduction}

There are so many studies developed on the oscillation of differential equations and difference equations in the past two decades. However, there are very few studies investigating the oscillatory behavior of difference equations with continuous variables. The readers are referred to [1] for the fundamentals of the oscillation theory of differential equations and [2]-[9] for fundamental results on difference equations with continuous arguments. In this paper, we study oscillatory behavior of a neutral difference equation of the form

$$
\nabla_{\alpha}[x(t)-r(t) x(t-\kappa)]+p(t) x(t-\tau)-q(t) x(t-\sigma)=0,
$$

where $t \geq t_{0}$ and $t$ travels through reals, $r \in C\left(\left[t_{0}-\alpha, \infty\right), \mathbb{R}^{+}\right), p, q \in$ $C\left(\left[t_{0}, \infty\right), \mathbb{R}^{+}\right), \kappa>\alpha>0, \tau>\sigma>\alpha$. Here, $\nabla_{\alpha}$ denotes the backward difference operator with the step $\alpha$, that is, $\nabla_{\alpha} x(t)=x(t)-x(t-\alpha)$ for $t \geq t_{0}$. For convenience in the paper, we set

$$
\beta:=\left\{\begin{array}{ll}
\tau, & r \equiv 0 \\
\max \{\kappa+\alpha, \tau\}, & r \not \equiv 0
\end{array} \text { and } \gamma:= \begin{cases}\sigma, & r \equiv 0 \\
\min \{\kappa, \sigma\}, & r \not \equiv 0 .\end{cases}\right.
$$

A function $x \in C\left(\left[t_{0}-\beta, \infty\right), \mathbb{R}\right)$ is called a solution of (1) if $x$ satisfies (1) on $\left[t_{0}, \infty\right)$. As is customary, a solution of (1) is called oscillatory if it

Received November 14, 2008.

2000 Mathematics Subject Classification. 39A10.

Key words and phrases. continuous variable, neutral difference equations, oscillation, positive and negative coefficients. 
has arbitrary large zeros; otherwise, such a solution is called nonoscillatory. Throughout the work, eventually trivial solutions of (1) are out of our interest.

\section{Main results}

For an arbitrary continuous function $f$ denote the $n$-th minimized function by

$$
f^{(n)}(t):= \begin{cases}f(t), & n=0 \\ \min \left\{f^{(n-1)}(\eta): t-\alpha \leq \eta \leq t\right\}, & n \in \mathbb{N}\end{cases}
$$

and let $h(t):=p(t)-q(t-\tau+\sigma)$.

Before stating our main results, we need to give some lemmas as follows. We start with the following lemma which is an extension of [5, Lemma 1].

Lemma 2.1. Assume that

$$
h(t) \geq 0(\not \equiv 0) \quad \text { and } \quad \int_{t-\alpha}^{t} r(\eta) \mathrm{d} \eta+\int_{t-\tau+\sigma}^{t} q(\eta) \mathrm{d} \eta \leq \alpha
$$

hold for all sufficiently large $t$. Let $x$ be an eventually positive solution of (1). Then the companion function of $x$ given by

$z_{x}(t):=\int_{t-\alpha}^{t} x(\eta) \mathrm{d} \eta-\int_{t-\alpha}^{t} r(\eta) x(\eta-\kappa) \mathrm{d} \eta-\int_{t-\tau+\sigma}^{t} q(\eta) x(\eta-\sigma) \mathrm{d} \eta, t \geq t_{0}+\beta$ satisfies

$$
z_{x}^{\prime} \leq 0(\not \equiv 0), \quad z_{x}>0
$$

on a subhalfline of $\left[t_{0}, \infty\right)$. Moreover,

$$
\nabla_{\alpha} z_{x}(t)+h^{(1)}(t) z_{x}(t-\tau) \leq 0
$$

holds on a subhalftine of $\left[t_{0}, \infty\right)$.

Proof. Let $t_{1} \geq t_{0}$ satisfy (2) for all $t \geq t_{1}$. Using the fact that $x$ is a solution of (1), we have

$$
\text { (6) } \begin{aligned}
z_{x}^{\prime}(t) & =\nabla_{\alpha}[x(t)-r(t) x(t-\kappa)]-q(t) x(t-\sigma)+q(t-\tau+\sigma) x(t-\tau) \\
& =-h(t) x(t-\tau) \leq 0(\not \equiv 0)
\end{aligned}
$$

for all $t \geq t_{2}$, where $t_{2} \geq t_{1}+\tau$. Hence, there exists $t_{3} \geq t_{2}$ such that $z_{x}$ is of constant sign on $\left[t_{3}, \infty\right)$. We claim that $z_{x}$ is of positive sign. Assume the contrary that $z_{x}(t) \leq 0$ holds for all $t \geq t_{3}$. Then, there exist $t_{4} \geq t_{3}$ and a constant $\mu>0$ satisfying $z_{x}(t)<-\mu$ for all $t \geq t_{4}$. Let $\phi:\left[t_{4}, \infty\right) \rightarrow \mathbb{R}$ be the function satisfying the mean value

$$
\alpha x(\phi(t))=\int_{t-\alpha}^{t} x(\eta) \mathrm{d} \eta
$$


for all $t \geq t_{4}$. Note that $\gamma \geq \alpha$ and $t-\alpha \leq \phi(t) \leq t$ for all $t \geq t_{4}$. In view of the definition of $z_{x}$ in (3), we obtain

$$
\begin{aligned}
\alpha x(\phi(t)) & <-\mu+\left(\int_{t-\alpha}^{t} r(\eta) \mathrm{d} \eta+\int_{t-\tau+\sigma}^{t} q(\eta) \mathrm{d} \eta\right) \max _{t-\beta \leq \eta \leq t-\gamma} x(\eta) \\
& \leq-\mu+\alpha \max _{t-\beta-\alpha \leq \eta \leq t} x(\phi(\eta))
\end{aligned}
$$

for all $t \geq t_{4}$. By the well-known result [1, Lemma 1.5.4], (7) yields a contradiction that $x \circ \phi$ can not be a positive function. This proves $z_{x}>0$ on $\left[t_{3}, \infty\right)$, that is, (4) holds. Integrating (6) over $[t-\alpha, t]$, we get

$$
\nabla_{\alpha} z_{x}(t)+\int_{t-\alpha}^{t} h(\eta) x(\eta-\tau) \mathrm{d} \eta=0,
$$

or

$$
\nabla_{\alpha} z_{x}(t)+h^{(1)}(t) \int_{t-\alpha}^{t} x(\eta-\tau) \mathrm{d} \eta \leq 0
$$

for all $t \geq t_{3}$. Taking (3) into account, we see that

$$
\int_{t-\alpha}^{t} x(\eta) \mathrm{d} \eta \geq z_{x}(t)
$$

holds for all $t \geq t_{4}$, where $t_{4} \geq t_{3}+\tau$. Substituting (9) into (8), we see that (5) holds on $\left[t_{4}, \infty\right)$. Hence, the proof is completed.

Now, we state the following result extracted from [3]:

Lemma 2.2. Consider the following delay differential equation

$$
x^{\prime}(t)+A(t) x(t-\rho)=0,
$$

where $\rho>0$ and $A \in C\left(\left[t_{0}, \infty\right), \mathbb{R}^{+}\right)$, and the corresponding delay differential inequality

$$
x^{\prime}(t)+A(t) x(t-\rho) \leq 0 .
$$

(10) possesses eventually positive solutions if and only if so does (11).

We give the following comparison theorem:

Theorem 2.1. Assume that (2) holds for all sufficiently large t. If every solution of

$$
y^{\prime}(t)+\frac{1}{\alpha} h^{(1)}(t) y(t-\tau+\alpha)=0
$$

is oscillatory, then every solution of (1) is oscillatory.

Proof. Assume the contrary that (1) has nonoscillatory solution $x$. Since (1) is linear, there is no loss in assuming $x$ as an eventually positive solution. Then, 
we have by Lemma 2.1 that $z_{x}$ introduced in (3) is nonincreasing. Let $t_{1} \geq t_{0}$ satisfy $(2), x(t)>0, z_{x}^{\prime}(t) \leq 0(\not \equiv 0)$ and $z_{x}(t)>0$ for all $t \geq t_{1}$. Now, we set

$$
y(t):=\int_{t-\alpha}^{t} z_{x}(\eta) \mathrm{d} \eta
$$

for $t \geq t_{2}$, where $t_{2} \geq t_{1}+\alpha$, then we have

$$
y^{\prime}(t)=\nabla_{\alpha} z_{x}(t) \leq 0
$$

for all $t \geq t_{2}$. Since $z_{x}$ is nonincreasing and positive on $\left[t_{2}, \infty\right)$, we deduce from (13) that

$$
0<\frac{1}{\alpha} y(t) \leq z_{x}(t-\alpha)
$$

holds for all $t \geq t_{2}$. Considering (5), (14) and (15), we get that

$$
y^{\prime}(t)+\frac{1}{\alpha} h^{(1)} y(t-\tau+\alpha) \leq 0,
$$

which indicates that the corresponding differential equation (12) has an eventually positive solution by Lemma 2.2. This contradiction completes the proof.

The following corollary allows us to test oscillatory behavior of all solutions of (1). For convenience, we introduce

$$
\bar{h}:=\limsup _{t \rightarrow \infty} \frac{1}{\alpha} \int_{t-\tau+\alpha}^{t} h^{(2)}(\eta) \mathrm{d} \eta \quad \text { and } \quad \underline{h}:=\liminf _{t \rightarrow \infty} \frac{1}{\alpha} \int_{t-\tau+\alpha}^{t} h^{(2)}(\eta) \mathrm{d} \eta .
$$

Corollary 2.1. Assume that (2) holds for all sufficiently large $t$. If

$$
\bar{h}>1 \quad \text { or } \quad \underline{h}>\frac{1}{\mathrm{e}}
$$

or

$$
\underline{h} \leq \frac{1}{\mathrm{e}} \quad \text { and } \quad \bar{h}>1-\frac{1-\underline{h}-\sqrt{1-2 \underline{h}-\underline{h}^{2}}}{2}
$$

holds, then every solution of (1) is oscillatory.

Proof. (12) can not have eventually positive solutions under either one of the conditions (16) or (17).

Now, we introduce

$$
\Lambda(t):=\{\lambda>0: 1-\lambda h(s)>0 \text { for all } s \geq t\}
$$

and

$$
\tau_{1}:=\left\lfloor\frac{\tau}{\alpha}\right\rfloor,
$$

where $\lfloor\cdot\rfloor$ denotes the lowest integer function. 
Corollary 2.2. Assume that (2) holds for all sufficiently large $t$, and

$$
\lim \sup _{t \rightarrow \infty} h(t)>0
$$

holds. Furthermore, assume that $\tau_{1} \in \mathbb{N}$ and

$$
\limsup _{t \rightarrow \infty} \sup _{\lambda \in \Lambda(t)}\left(\lambda \prod_{i=1}^{\tau_{1}-1}\left[1-\lambda h^{(2)}(t-i \alpha)\right]\right)<1 .
$$

Then every solution of (1) is oscillatory.

Proof. The proof follows from the papers $[2,4,7]$.

\section{Iterative results}

In this section, we improve the results of the previous section by iteration.

We first improve Lemma 2.1 by introducing $\tau_{2}:=\lfloor(\tau-\sigma) / \alpha\rfloor$ and the recursion

$$
h_{n}(t):= \begin{cases}1, & n=0 \\ r^{(1)}(t) h_{n-1}(t-\kappa)+\sum_{j=0}^{\tau_{2}-1} q^{(1)}(t-j \alpha) h_{n-1}(t-j \alpha-\sigma), & n \in \mathbb{N} .\end{cases}
$$

Lemma 3.1. Assume that $\tau_{2} \in \mathbb{N}$ and all conditions of Lemma 2.1 are held. Then, for each $n \in \mathbb{N}$, there exists a halfline $J_{n} \subset\left[t_{0}, \infty\right)$ such that $z_{x}$ introduced in (3) satisfies

$$
\nabla_{\alpha} z_{x}(t)+h^{(1)}(t) \sum_{i=0}^{n} h_{i}(t-\tau) z_{x}(t-\tau) \leq 0
$$

for all $t \in J_{n}$.

Proof. Assume that conclusions of Lemma 2.1 hold for all $t \geq t_{1}$, where $t_{1} \geq t_{0}$. Let $h_{n}$ function in (18) be defined for $t \geq t_{1}+n \beta$, where $n \in \mathbb{N}$. We see from (3) for $t \geq t_{1}$ that

$$
\begin{aligned}
\int_{t-\alpha}^{t} x(\eta) \mathrm{d} \eta= & z_{x}(t)+\int_{t-\alpha}^{t} r(\eta) x(\eta-\kappa) \mathrm{d} \eta+\int_{t-\tau+\sigma}^{t} q(\eta) x(\eta-\sigma) \mathrm{d} \eta \\
\geq & z_{x}(t)+r^{(1)}(t) \int_{t-\alpha}^{t} x(\eta-\kappa) \mathrm{d} \eta \\
& +\sum_{j=0}^{\tau_{2}-1} q^{(1)}(t-j \alpha) \int_{t-(j+1) \alpha}^{t-j \alpha} x(\eta-\sigma) \mathrm{d} \eta
\end{aligned}
$$

holds. Now, we prove by induction that

$$
\int_{t-\alpha}^{t} x(\eta) \mathrm{d} \eta \geq \sum_{i=0}^{m} h_{i}(t) z_{x}(t)
$$


holds for all $t \geq t_{1}+m \beta$ and $m=0,1, \ldots, n$. Clearly, the above inequality holds for $m=0$ trivially from (9). Suppose that (21) holds for $m=n-1$ and all $t \geq t_{1}+(n-1) \beta$. Now, we show that (21) also holds for $m=n$ and all $t \geq t_{1}+n \beta$. Consequently, from (20), (21) and the nonincreasing nature of $z_{x}$, we have

$(22)$

$$
\begin{aligned}
& \int_{t-\alpha}^{t} x(\eta) \mathrm{d} \eta \\
\geq & z_{x}(t)+r^{(1)}(t) \sum_{i=0}^{n-1} h_{i}(t-\kappa) z_{x}(t-\kappa) \\
& +\sum_{i=0}^{n-1} \sum_{j=0}^{\tau_{2}-1} q^{(1)}(t-j \alpha) h_{i}(t-j \alpha-\sigma) z_{x}(t-j \alpha-\sigma) \\
\geq & \left(1+\sum_{i=0}^{n-1}\left[r^{(1)}(t) h_{i}(t-\kappa)+\sum_{j=0}^{\tau_{2}-1} q^{(1)}(t-j \alpha) h_{i}(t-j \alpha-\sigma)\right]\right) z_{x}(t) \\
= & \left(1+\sum_{i=0}^{n-1} h_{i+1}(t)\right) z_{x}(t)=\sum_{i=0}^{n} h_{i}(t) z_{x}(t)
\end{aligned}
$$

for all $t \geq t_{1}+n \beta$. Substituting (22) into (8), we obtain that (19) holds on $J_{n}:=\left[t_{1}+n \beta, \infty\right)$. This completes the proof of the lemma.

Remark 3.1. Note that Lemma 2.1 is a particular case of Lemma 3.1 with $n=0$.

Now, we give the following result which is an extension of Theorem 2.1:

Theorem 3.1. Assume that assumptions of Lemma 3.1 are held. If there exists $n_{0} \in \mathbb{N}$ such that every solution of

$$
y^{\prime}(t)+\frac{1}{\alpha} h^{(1)}(t) \sum_{i=0}^{n_{0}} h_{i}(t-\tau) y(t-\tau+\alpha)=0
$$

is oscillatory, then every solution of (1) is also oscillatory.

Proof. Since all assumptions of Lemma 2.1, Theorem 2.1 and Lemma 3.1 are held. Considering (14), (15), and substituting (13) into (19), we see that the corresponding equation (23) has an eventually positive solution by Lemma 2.2, and this contradiction completes the proof.

For convenience, we need to introduce the followings:

$$
\bar{h}(n):=\limsup _{t \rightarrow \infty} \frac{1}{\alpha} \int_{t-\tau+\alpha}^{t} h^{(2)}(\eta) \sum_{i=0}^{n} h_{i}(\eta-\tau) \mathrm{d} \eta,
$$




$$
\underline{h}(n):=\liminf _{t \rightarrow \infty} \frac{1}{\alpha} \int_{t-\tau+\alpha}^{t} h^{(2)}(\eta) \sum_{i=0}^{n} h_{i}(\eta-\tau) \mathrm{d} \eta .
$$

Corollary 3.1. Assume that assumptions of Lemma 3.1 are held. If there exists $n_{0} \in \mathbb{N}$ satisfying

$$
\bar{h}\left(n_{0}\right)>1 \quad \text { or } \quad \underline{h}\left(n_{0}\right)>\frac{1}{\mathrm{e}}
$$

or

$$
\underline{h}\left(n_{0}\right) \leq \frac{1}{\mathrm{e}} \quad \text { and } \quad \bar{h}\left(n_{0}\right)>1-\frac{1-\underline{h}\left(n_{0}\right)-\sqrt{1-2 \underline{h}\left(n_{0}\right)-\underline{h}\left(n_{0}\right)^{2}}}{2},
$$

then every solution of (1) is oscillatory.

Corollary 3.2. Assume that assumptions of Lemma 3.1 are held. If $\underline{h}(\infty)>$ $1 / \mathrm{e}$ or $\bar{h}(\infty)>1$ holds, then every solution of (1) is oscillatory.

Proof. The claim follows by Corollary 3.1 , since $\underline{h}(n)$ is nondecreasing and $\underline{h}(\infty)>1 /$ e or $\bar{h}(\infty)>1$ holds.

The following theorem is useful for testing oscillatory behavior of (1) when $r$ and $q$ are nonincreasing functions.

Theorem 3.2. Assume that assumptions of Lemma 3.1 are held. Moreover, $r$ and $q$ are nonincreasing. If there exists $n_{0} \in \mathbb{N}$ such that

$$
\liminf _{t \rightarrow \infty} \frac{1}{\alpha} \int_{t-\tau+\alpha}^{t} h^{(2)}(\eta) \sum_{i=0}^{n_{0}}\left[r(\eta)+\tau_{2} q(\eta)\right]^{i} \mathrm{~d} \eta>\frac{1}{\mathrm{e}}
$$

or

$$
\limsup _{t \rightarrow \infty} \frac{1}{\alpha} \int_{t-\tau+\alpha}^{t} h^{(2)}(\eta) \sum_{i=0}^{n_{0}}\left[r(\eta)+\tau_{2} q(\eta)\right]^{i} \mathrm{~d} \eta>1
$$

holds, then every solution of (1) is oscillatory.

Proof. Since, $r$ and $q$ are nonincreasing, we eventually have

$$
\begin{aligned}
& h_{0}(t)=1, \\
& h_{1}(t)=r^{(1)}(t)+\sum_{j=0}^{\tau_{2}-1} q^{(1)}(t-j \alpha) \geq r(t)+\tau_{2} q(t),
\end{aligned}
$$

and in general, we can see that

$$
h_{n}(t) \geq\left[r(t)+\tau_{2} q(t)\right]^{n}, \quad n \in \mathbb{N}
$$

holds. Applying Corollary 3.1, we see that every solution of (1) is oscillatory.

Now, we consider the following scalar equation

$$
\nabla_{\alpha}[x(t)-r x(t-\kappa)]+p x(t-\tau)-q x(t-\sigma)=0 .
$$


Theorem 3.3. Assume that

$$
\begin{aligned}
\tau_{2} & =\left\lfloor\frac{\tau-\sigma}{\alpha}\right\rfloor \in \mathbb{N}, \\
r & \geq 0, \quad p>q \geq 0, \\
0 & \leq \alpha r+q(\tau-\sigma) \leq \alpha
\end{aligned}
$$

hold. If

$$
\frac{(\tau-\alpha)(p-q)}{\alpha\left(1-\left(r+\tau_{2} q\right)\right)}>\frac{1}{\mathrm{e}}
$$

holds, then every solution of (24) is oscillatory.

Proof. As is estimated in Theorem 3.2, we have $h_{n}(t)=\left[r+\tau_{2} q\right]^{n}$ for $n \in \mathbb{N}$. Now, we consider the following two possible cases:

Case 1. $r+q \tau_{2} \geq 1$. In this case, we see that

$$
\begin{aligned}
\underline{h}(n) & =\frac{(\tau-\alpha)(p-q)}{\alpha} \sum_{i=0}^{n}\left[r+\tau_{2} q\right]^{i} \geq \frac{(\tau-\alpha)(p-q)}{\alpha} \sum_{i=0}^{n} 1 \\
& =\frac{(\tau-\alpha)(p-q)}{\alpha}(n+1)
\end{aligned}
$$

which implies $\underline{h}(\infty)=\infty$, therefore Corollary 3.2 is applicable.

Case 2. $0 \leq r+q \tau_{2}<1$. In this case, we see that

$$
\underline{h}(n)=\frac{(\tau-\alpha)(p-q)}{\alpha} \sum_{i=0}^{n}\left[r+\tau_{2} q\right]^{i}
$$

holds. Therefore, we obtain

$$
\underline{h}(\infty)=\frac{(\tau-\alpha)(p-q)}{\alpha\left(1-\left(r+\tau_{2} q\right)\right)}>\frac{1}{\mathrm{e}} .
$$

We complete the proof by considering (25) and applying Corollary 3.2.

\section{References}

[1] I. Gyori and G. Ladas, Oscillation Theory of Delay Difference Equations with Applications, Oxford Mathematical Monographs. Oxford Science Publications. The Clarendon Press, Oxford University Press, New York, 1991.

[2] X. Li and D. Zhu, Comparison theorems of oscillation and nonoscillation for neutral difference equations with continuous arguments, Commun. Pure Appl. Anal. 2 (2003), no. 4, 579-589.

[3] C. G. Philos, Oscillation for first order linear delay differential equations with variable coefficients, Funkcial. Ekvac. 35 (1992), no. 2, 307-319.

[4] J. H. Shen, Comparison theorems for oscillations of difference equations with continuous variables, Kexue Tongbao (Chinese) 41 (1996), no. 16, 1441-1444.

[5] B. G. Zhang, Oscillations of a class of difference equations with continuous arguments, Appl. Math. Lett. 14 (2001), no. 5, 557-561.

[6] B. G. Zhang and B. M. Liu, Linearized oscillation theorems for certain nonlinear difference equations with continuous arguments, Math. Comput. Modelling 30 (1999), no. 1-2, $89-96$. 
[7] B. G. Zhang, J. Yan, and S. K. Choi, Oscillation for difference equations with continuous variable, Comput. Math. Appl. 36 (1998), no. 9, 11-18.

[8] Y. Zhang and J. Yan, Oscillation criteria for difference equations with continuous arguments, Acta Math. Sinica 38 (1995), no. 3, 406-411.

[9] Y. Zhou, Oscillation of difference equations with variable coefficients and continuous arguments, Economic Math. 13 (1996), no. 1, 86-89.

B. KARPUZ

Department of Mathematics

Faculty of Sciences And ARts

ANS CAmpus, 03200, Afyonkarahisar, Turkey

E-mail address: bkarpuz@gmail.com

ÖZKAN ÖCALAN

Department of Mathematics

FACULTY OF SCIENCES AND ARTS

ANS Campus, 03200, Afyonkarahisar, Turkey

E-mail address: ozkan@aku.edu.tr 